\title{
Detector upgrade for the KLOE2 experiment:The Inner Tracker
}

\author{
Paolo Branchini ${ }^{1}$ on behalf of the KLOE2 collaboration \\ Affiliation INFN RomaTRE \\ Via Della Vasca Navale 84,Roma, Italy. \\ E-mail: Paolo.Branchinieroma3.infn. it
}

\section{G. Bencivenni ${ }^{a}$, S. Cerioni ${ }^{a}$, D. Dominici ${ }^{a}$, M. Gatta $^{a}$, S. Lauciani ${ }^{a}$, G. Pileggi ${ }^{a}$, M Pistilli \\ ${ }^{a}$ Laboratori Nazionali di Frascati, Frascati (Rm) Italy.}

Abstract

\begin{abstract}
A low mass, fully cylindrical and dead region free GEM detector is under development as Inner Tracker for the KLOE experiment upgrade at the DA $\Phi$ NE facility in Frascati. The proposed detector will play a crucial role in the study of the $\mathrm{K}_{\mathrm{s}}$ decays and interferometry. The main physics requirements are: good detector spatial resolution $\sigma_{\mathrm{r} \phi}$ about $200 \mu \mathrm{m}$ and $\sigma_{\mathrm{z}}$ about 500 $\mu \mathrm{m}$ and a very low material budget inside the active area $1.5 \%$ of radiation length $\left(\mathrm{X}_{0}\right)$ for the whole detector. The detector consists of five layers of cylindrical triple-GEM detectors (CGEM), covering the space from the beam pipe to the inner cylinder of the KLOE main Drift Chamber. Each layer is realized inserting one into the other the required structure made of 50 $\mu \mathrm{m}$ polyimide foils. The final result is a very light detector with a $0.2 \%$ of $\mathrm{X}_{0}$ per tracking layer inside the active area. After the successful construction and test of a small size prototype $90 \mathrm{~mm}$ diameter, $250 \mathrm{~mm}$ length, a full scale prototype (300 $\mathrm{mm}$ diameter, $352 \mathrm{~mm}$ length) of the first layer of the Inner Tracker has been designed and built in all details. We report about the design, construction and some preliminary test of such a large prototype that open the way for a new and competitive category of ultra-light micro pattern gas vertex detector.
\end{abstract}

\footnotetext{
$1 \quad$ Speaker
} 


\section{Introduction}

The KLOE experimental activity is planned to continue at the DA $\Phi N E \mathrm{e}^{+} \mathrm{e}^{+}$machine upgraded in luminosity and energy. The challenge is to improve the systematics at the level demanded by the increase of the integrated luminosity to $\sim 50 \mathrm{fb}^{-1}$ in 3-4 years of running. The vast physics program, mainly concerning $K_{s}, \eta$, kaon interferometry, charged kaon decays, is focused on events produced close to the interaction point (IP), requiring an optimization of the detection for low momentum tracks coming from the IP and from the Kaon decays. Besides a normal revision of some subsystem (Drift Chamber, FEE, DAQ, online/offline systems) the evolution of the KLOE apparatus foresees the upgrade of the scintillating fiber calorimeter and the insertions of three new devices: a crystal calorimeter placed in front of the Quads, a $\gamma \gamma$ tagger and an Inner Tracker (IT). The $\gamma \gamma$ tagger is devoted to detect small angles interactions with a technology of $\mathrm{Si}$ micro-strips and a plastic scintillator hodoscope. The Inner Tracker exploits a novel technology of fully cylindrical GEM (Gas Electron Multiplier [1] detectors. It is composed by five concentric layers providing a point space 2-D measurement of the track. Each layer is a tripleGEM chamber with cathode and anode made of thin polyimide foils, in order to reduce the material budget. The result is an ultra-light and dead-zone free detector representing a completely new step in the development of tracking devices with gas detectors.

\section{The Inner Tracker}

The Inner Tracker is composed by five independent tracking layers (L1-L5), in order to achieve a good track reconstruction. The innermost layer will be placed at $15 \mathrm{~cm}$ from the beam line, corresponding to $20 \tau_{\mathrm{s}}$ in order not to spoil the $\mathrm{K}_{\mathrm{l}} \mathrm{K}_{\mathrm{s}}$ interference. The outermost layer will be placed at $25 \mathrm{~cm}$ from the beam line, just inside the internal wall of the Drift Chamber.

\section{Simulation results}

The Inner Tracker contribution to the overall tracking capability has been simulated implementing the new device in the existing reconstruction algorithm of KLOE. Preliminary results for a pion track from a $\mathrm{K}_{\mathrm{s}} \rightarrow \pi^{+} \pi^{-}$decay are shown in Table [1].

\begin{tabular}{|l|l|l|l|l|}
\hline & $\Delta \mathrm{x} @ \mathrm{pca}$ & $\Delta \mathrm{z} @ \mathrm{pca}$ & $\Delta \mathrm{p}_{\mathrm{x}} @ \mathrm{pca}$ & $\Delta \mathrm{x} @ \mathrm{vtx}$ \\
\hline IT & $0.6 \mathrm{~mm}$ & $0.9 \mathrm{~mm}$ & $1.2 \mathrm{MeV} / \mathrm{c}$ & $1.9 \mathrm{~mm}$ \\
\hline No IT & $1.7 \mathrm{~mm}$ & $2.2 \mathrm{~mm}$ & $1.6 \mathrm{MeV} / \mathrm{c}$ & $4.9 \mathrm{~mm}$ \\
\hline
\end{tabular}

Table 1: Reconstruction parameters for a pion track with and without the Inner Tracker.

The reconstruction variables in the first three columns indicate the difference respectively of the $\mathrm{x}$ coordinate, $\mathrm{z}$ coordinate and $\mathrm{x}$ momentum component between the point of closest approach (pca) of the track with respect to the vertex and the vertex itself; the fourth column indicates the sigma of the difference between the $\mathrm{x}$ coordinate of the reconstructed and Monte Carlo vertex. Although the algorithm is not yet optimized for the new geometry, it is clear how the information from the IT noticeably improve the reconstruction capability of the detector. 


\section{Inner Tracker motivations and requirements}

The insertion of an Inner Tracker in the present KLOE configuration is requested for the optimization of the reconstruction for the physics coming from the interaction region, namely for a fine vertex reconstruction of the $K_{s}, \eta$ and $\eta$ ' decay products.

The detector requirements are:

1. $\sigma_{\mathrm{r} \phi} \sim 200 \mu \mathrm{m}$ spatial resolution;

2. $5 \mathrm{kHz} / \mathrm{cm}^{2}$ rate capability;

3. $1.5 \% \mathrm{X}_{0}$ overall material budget.

While the first two requirements can be easily accomplished by a standard GEM detector, we have developed the technology of fully cylindrical GEM in order to fulfill the very stringent requirement on the material budget, needed to minimize the multiple scattering effect for lowmomentum tracks.

A cylindrical triple-GEM detector (C-GEM) is thus obtained inserting one into the other the five cylindrical electrodes defining the gaps with a pattern of $3 / 2 / 2 / 2 \mathrm{~mm}$ respectively for the Drift/Transfer1/Transfer2/Induction Figure 1.

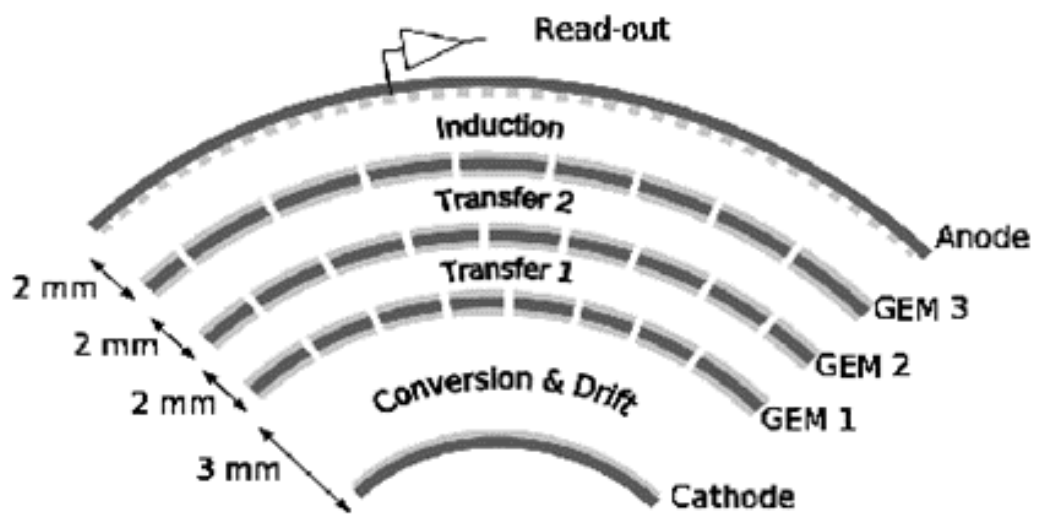

Figure 1: Scheme of a cylindrical triple-GEM detector.

All the electrodes are realized with thin polyimide foils with a copper clad, resulting in an ultralight detector. Moreover all the support mechanics is placed outside from the active area, at the edges of the cylinder, allowing the construction of a detector practically free of dead zones. In fact the structural rigidity and the tension of the GEMs is achieved by stretching the detector along its longitudinal axis. All the electrodes are realized with thin polyimide foils with a copper clad, resulting in an ultra-light detector.

\section{The small size prototype}

In 2006 [2] a small size prototype of cylindrical GEM has been built recycling GEM foils designed for the LHCb Muon System [3]. Due to the dimensions of the foils (20x24 $\mathrm{cm}^{2}$ active area) the chamber had a diameter of only $9 \mathrm{~cm}$. This prototype represented the very first test of the novel idea of fully cylindrical GEM detectors, and several construction solutions have been successfully exploited, as the vacuum-bag technique for the gluing of the electrodes. The scheme of the detector was that of a standard triple-GEM with $3 / 2 / 2 / 2 \mathrm{~mm}$ gaps (respectively for the Drift/Transfer1/Transfer2/Induction). Both the cathode and the anode have been obtained as single polyimide foils $(50 \mu \mathrm{m}$ thickness). Hence the readout had no segmentation and the chamber could only be tested in current mode. Nevertheless it has been operated with a 
$\mathrm{Ar} / \mathrm{CO}_{2}(70 / 30)$ gas mixture, and tested with a X-ray gun of $6 \mathrm{keV}$ energy. The basic idea of the detector has been proved valid and the characteristic plots of the electron transparency as a function of the various fields have been obtained, resulting in good agreement with the reference values found in literature. A gain plot is shown in figure 2. A gain value of 10000 without any discharge or leakage currents has been reached.

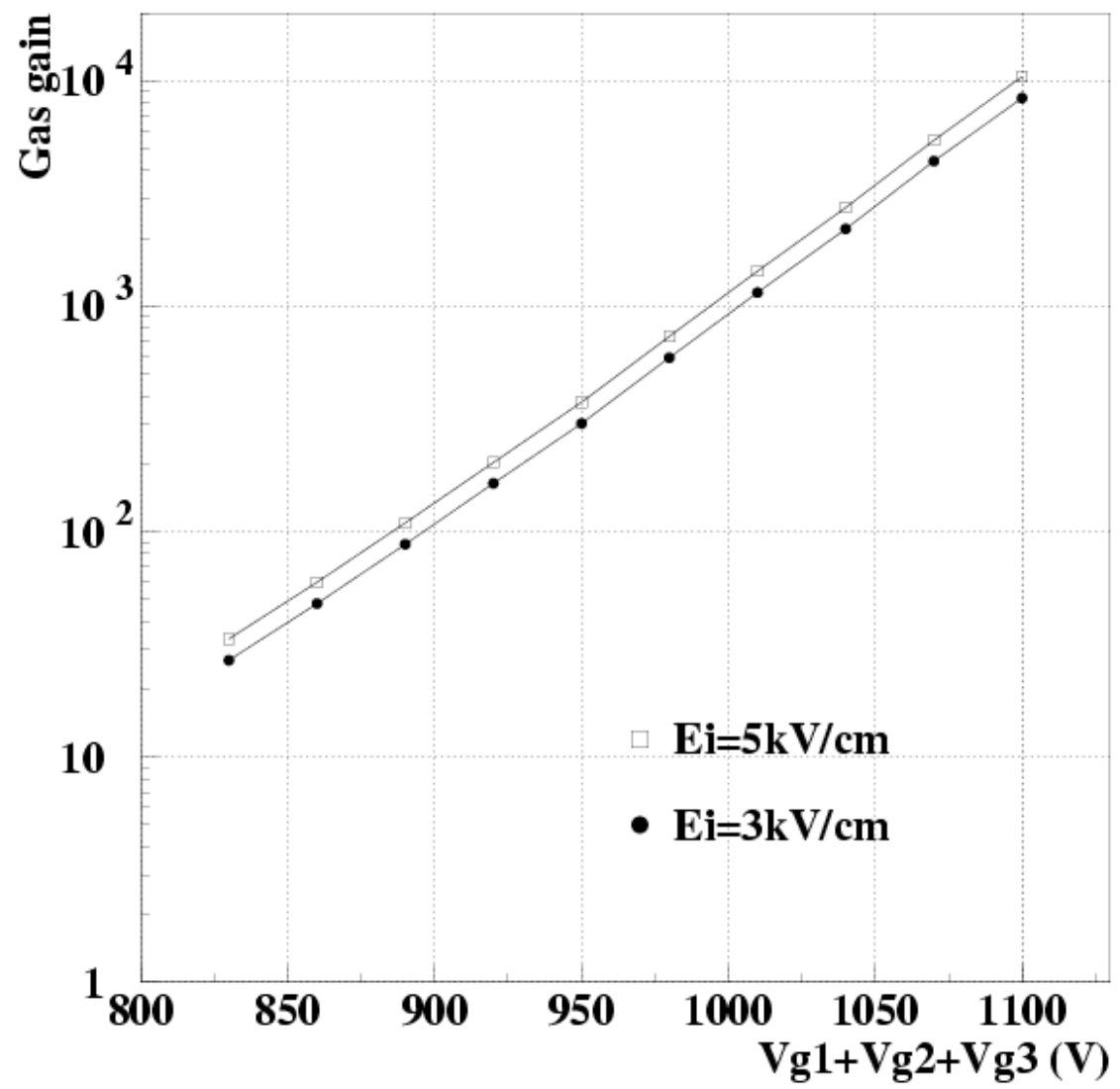

Figure 2: The gas gain measured for a $\mathrm{Ar} / \mathrm{Co}_{2}$ mixture at two different values of the induction field

\section{The full scale prototype}

in 2007 we started the construction of a new prototype with larger dimensions, similar to those of the Layer1 of the final KLOE Inner Tracker: it has the same diameter of $300 \mathrm{~mm}$ but a reduced active length of $352 \mathrm{~mm}$ (instead of the foreseen $600 \mathrm{~mm}$ ), due to the present limitation on the GEM foil production technique. For the same reason a single cylindrical electrode $\left(352 \times 960 \mathrm{~mm}^{2}\right)$ has been obtained as a join of three identical GEM foils $(352 \times 320$ $\mathrm{mm}^{2}$ ). The foils have been glued with an overlap region $3 \mathrm{~mm}$ wide where the copper has been completely etched. The cathode is realized as a unique polyimide foil $(100 \mu \mathrm{m})$ with a copper clad $(18 \mu \mathrm{m})$ on the internal face. The anode is realized as a join of 3 foils, made of polyimide, with a read-out segmentation composed by a 1-dimensional set of strips along the axis of the cylinder, for the r $\phi$ coordinate read-out. The 1538 strips have a $650 \mu \mathrm{m}$ pitch. 


\section{The C-GEM building procedure}

The following are the steps performed to obtain a cylindrical GEM foil:

1. an epoxy adhesive (typically Araldite) is distributed along one edge ( $3 \mathrm{~mm}$ wide) of the foil;

2. the foil is rolled on an Aluminum mould coated with a very precise $400 \mu \mathrm{m}$ thick machined Teflon film, to have a non-stick, low-friction surface;

3. the cylinder is enveloped in a vacuum bag and the vacuum is obtained with a Venturi system, providing a high $\left(1 \mathrm{~kg} / \mathrm{cm}^{2}\right)$ and uniform pressure throughout the surface of the cylinder;

4. the foil is easily extracted from the mould, thanks to its Teflon surface, and a cylindrical GEM is obtained;

5. with the same technique the cathode and anode foils are obtained as well.

\section{The cylindrical cathode}

The cathode is realized as a unique polyimide foil, $100 \mu \mathrm{m}$ thick, with a copper clad of $18 \mu \mathrm{m}$ on the internal side. All the support mechanics of the chamber is composed by annular flanges made of Permaglass placed at the edges of the cylinder. They account for the gas inlets and outlets and their thickness defines the distance between the various electrodes.

\section{The GEM foils}

The GEM foils have an area of $352 \times 320 \mathrm{~mm}^{2}$, being one of the largest ever built. For a safe detector operation the foil has independent high voltage sectors, in order to limit the capacitance and hence the energy released through the GEM hole in case of a discharge. Each foil has 20 sectors, with an area of about $56 \mathrm{~cm}^{2}$ meaning a width of $1.6 \mathrm{~cm}$ for the single sector. Three foils are preliminary joined together, providing a very large foil of 960x352 mm $\mathrm{mm}^{2}$. The planar gluing is executed on a table with the technique of vacuum bag. The glue is an epoxy adhesive and the overlaps regions are $3 \mathrm{~mm}$ wide.

\section{The anode read-out}

Also the anode is realized as a join of three foils. Each foil hosts the readout copper strips and the ground. The copper strips side and the ground side are placed on the opposite faces of two different polyimide foils, staggered and glued together. In this way the readout strips have no discontinuity and the pitch value is preserved across the overlap region. In order to carry out the signals, the strips end-up in polyimide flaps as shown in figure 3, each grouping 32 strips, where the FEE is plugged with ZIF connectors. The pitch of the strips is $650 \mu \mathrm{m}$ in the readout zone, thus providing, when equipped with a digital readout, a $\sigma \sim 200 \mu \mathrm{m}$ spatial resolution, fulfilling the detector requirement in Section 4. In the flaps the pitch is reduced to $500 \mu \mathrm{m}$ in order to match the pitch of the connector.

\section{The front end electronics}

Two different solutions are foreseen to equip the prototype and the final detector. For the time being we are using a FEE based on the CARIOCA chip, initially developed for the MWPC of the LHCb Muon System, and then adapted to the GEM. It is a digital chip with 8 channels. In order to properly accomodate the chip to the prototype geometry two new boards have been designed and realized: a motherboard plugged directly to the detector with ZIF connectors, 
hosting the regulators and the I/O connectors; a daughterboard hosting two CARIOCA chips. Moreover 2 daughter boards are plugged on one motherboard, one on each side, for a total of 32 channels filling in a $2 \mathrm{~cm}$ wide space. For the final detector we are developing, in collaboration with the INFN-Bari group, a dedicated chip , providing amplification/shaping/discrimination for 64 channels with serial readout.

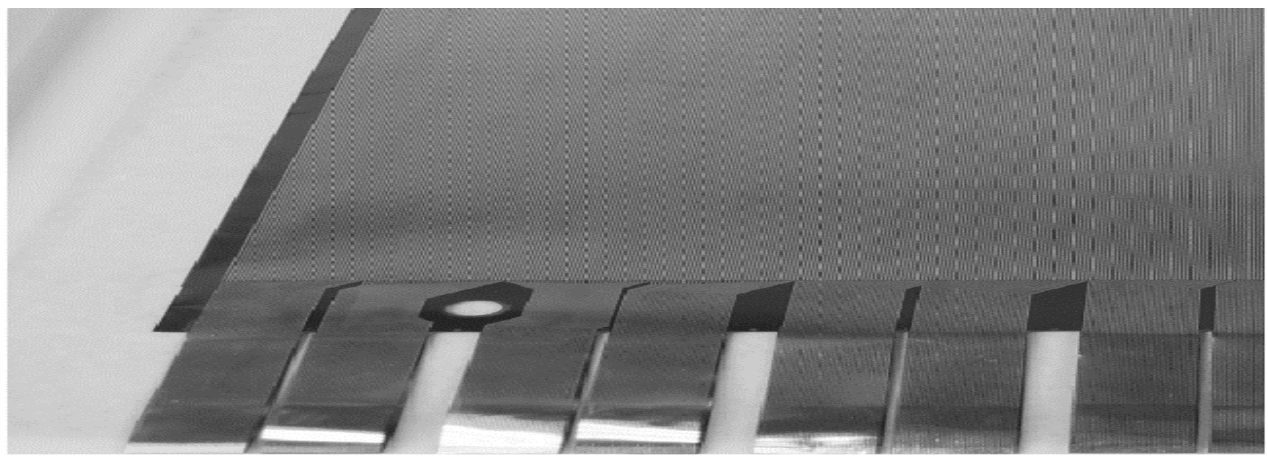

Figure 3: The anode with the strips for the readout and the flaps for the bonding of the FEE.

\section{Preliminary test results}

The chamber has been flushed with a $\mathrm{Ar} / \mathrm{CO}_{2}$ (70/30) gas mixture and operated up to an estimated gain of 5000. No leakage current has been observed, nor discharges. In the next months an extensive test program is foreseen, both with $\mathrm{X}$-rays, cosmic rays and test beams, in order to fully characterize the detector. A study on different gas mixtures (e.g. $\mathrm{Ar} / \mathrm{i}-\mathrm{C}_{4} \mathrm{H}_{10} / \mathrm{CF}_{4}$ ) is scheduled as well.

\section{Conclusions}

The KLOE detector is ready to take up the challenge given by the next $50 \mathrm{fb}^{-1}$ of integrated luminosity delivered by the upgraded DAFNE accelerator, in Frascati. Complex hardware improvements are planned both to reduce the systematics and to open new physics channels. In order to optimize the reconstruction capability for the decays near the interaction point a new Inner Tracker will be inserted inside the Drift Chamber. To fulfill the stringent requirement on the material budget of the IT $\left(<1.5 \mathrm{X}_{0}\right)$ we have developed a novel technique of fully cylindrical GEM detector. The mechanics has been designed so that no dead zone is present within the active area. The necessary rigidity has been obtained with a longitudinally stretch of the chamber. A prototype with dimensions similar to those of the final IT has been successfully built, overcoming several assembling difficulties. The positive results open the way for a new and valuable category of ultra-light Micro Pattern Gas Detectors for vertexing purpose.

\section{References}

[1] F. Sauli, Nucl.Instr.Meth. A386 (1997) 531.

[2] G.Bencivenni et al., Nucl.Instr.Meth. A572 (2007) 168.

[3] LHCb Muon System TDR, CERN LHCC 2001-010. 